\title{
Avaliação de tratamentos de sementes e inseticidas no solo para controle do percevejo-preto, Cyrtomenus mirabilis (Hemiptera: Cydnidae) em amendoim
}

\author{
Submetido - 30 jul. 2020 \\ Aprovado - 09 set. 2020 \\ Publicado - 14 out. 2020
}

http://dx.doi.org/10.17648/sas.v1i2.57

\begin{abstract}
Alessandro Martins Xavier (iD
Graduando em Agronomia - Unilago, São José do Rio Preto, SP, alessandro.saopaulo56@gmail.com.

Kauan Murilo Hernandes (D)

Graduando em Agronomia - Unifipa, Catanduva, SP, kauanmh@gmail.com.
\end{abstract}

Olavo Betiol (iD

Eng. Agrônomo, Mestrando em Agronomia - Departamento de Solos, FCAV/Unesp, Jaboticabal.

Denizart Bolonhezi ${ }^{(1)}$

Dr., Pesquisador Científico - IAC, Centro de Cana, Ribeirão Preto, SP, denizart@iac.sp.gov.br.

Ignácio José de Godoy

Dr., Pesquisador Científico - IAC, Centro de Grãos e Fibras, Campinas, SP, ijgodoy@iac.sp.gov.br.

Marcos Doniseti Michelotto (i)

Dr., Pesquisador Cientifico - Apta, Polo Centro Norte, Pindorama, SP, marcos.michelotto@sp.gov.br.

\begin{abstract}
RESUMO
Recentemente tem-se observado um aumento expressivo na ocorrência do ataque do percevejo-preto, Cyrtomenus mirabilis (Hemiptera: Cydnidae), em amendoim. Em função do hábito subterrâneo, seu controle é extremamente difícil. Até o momento não há inseticidas recomendados para seu controle. Assim, o objetivo do trabalho foi avaliar a eficiência de inseticidas no controle de ninfas e adultos do percevejo-preto e analisar o resíduo dos inseticidas nos grãos de amendoim. Para isso, foram instalados dois experimentos em área do Polo Centro Norte em Pindorama, SP, e no Centro de Cana do IAC em Ribeirão Preto, SP. Os experimentos foram em blocos casualizados com dez tratamentos, e quatro repetições. Ao final do ciclo do amendoim foi contabilizado o número de percevejos por trincheira e avaliado os danos causados pelos mesmos nos diferentes tratamentos. Em Pindorama a baixa ocorrência do inseto impediu as comparações. Já em Ribeirão Preto foi possível observar diferenças entre os tratamentos. Observou-se que os tratamentos Standak Top $\left(4,0 \mathrm{~mL} \mathrm{~kg}^{-1}\right.$ de sementes) e Standak Top (2,0 mL kg-1 de sementes) associado ao Regent Duo (500 mL ha-1) são os melhores tratamentos para controle e redução dos danos ocasionados pelo percevejo-preto; Os inseticidas nas doses e nas condições avaliadas não apresentam resíduo nos grãos de amendoim na colheita.
\end{abstract}

Palavras-chave: Arachis hypogeae L., Praga de solo; Resíduo de inseticida.

\section{Evaluation of seed treatments and at-plant soil insecticides for the control of burrower bug, Cyrtomenus mirabilis (Hemiptera: Cydnidae) in peanut}

\author{
ABSTRACT
}

Recently, a significant increase in the occurrence of the burrower bug, Cyrtomenus mirabilis (Hemiptera: Cydnidae), has been observed in peanuts. Due to the underground habit, its control is extremely difficult. So far, there are no insecticides recommended for its control. Thus, the objective of the work was to evaluate the efficiency of insecticides in the control of nymphs and adults of the burrower bug and to analyze the residue of the insecticides in the peanut kernels. 
For this, two experiments were installed at Apta Centro Norte in Pindorama, SP, and at Sugarcane Research Center / IAC in Ribeirão Preto, SP. Those experiments were in randomized blocks with ten treatments, and four replications. At the end of the peanut cycle, the number of insects per trench was counted and the damage caused by them in the different treatments was evaluated. In Pindorama, in order to the low occurrence no statistic analysis was done in terms of insect number. On the other hand, in Ribeirão Preto it was possible to observe differences between treatments. It was observed that the treatments Standak Top ( $4 \mathrm{~mL} \mathrm{~kg}^{-1}$ of seeds) and Standak Top ( $2 \mathrm{~mL} \mathrm{~kg}^{-1}$ of seeds) associated with Regent Duo (500 $\mathrm{mL} \mathrm{ha}^{-1}$ ) were the best treatments for controlling and reducing the damage caused by the $\mathrm{C}$. mirabilis. The insecticides in the doses and evaluated conditions do not present residues of insecticides in the peanut kernels at harvest.

Keywords: Arachis hypogeae L.; Soil pest; Pesticide residue.

\title{
Evaluación de tratamientos de semillas e insecticidas en el suelo para controlar chinches, Cyrtomenus mirabilis (Hemiptera: Cydnidae) en maní
}

\begin{abstract}
RESUMEN
Recientemente, se ha observado un aumento significativo en la ocurrencia del ataque del chinche, Cyrtomenus mirabilis (Hemiptera: Cydnidae), en el maní. Debido a su hábito subterráneo, su control es es muy complicado. Hasta el momento no hay insecticidas recomendados para su control. Por lo tanto, el objetivo del trabajo fue evaluar la eficiencia de los insecticidas en el control de las ninfas y adultos del insecto y analizar los residuos de los insecticidas en los granos de maní. Para esto, se instalaron dos experimentos en el área del Polo Centro Norte en Pindorama, SP, y en el Centro IAC en Cana en Ribeirão Preto, SP. LoS experimentos fueron en bloques al azar con diez tratamientos y cuatro repeticiones. Al final del ciclo de maní, se contó el número de chinches por zanja y se evaluó el daño causado por ellos en los diferentes tratamientos. En Pindorama, la baja ocurrencia del insecto impidió las comparaciones. En Ribeirão Preto fue posible observar diferencias entre tratamientos. Se observó que los tratamientos Standak Top (4 mL kg-1 de semillas) y Standak Top (2 mL $\mathrm{kg}^{-1} \mathrm{de}$ semillas) asociados con Regent Duo (500 ml ha-1) son los mejores tratamientos para controlar y reducir el daño causado por el chinche; Los insecticidas en las dosis y en las condiciones evaluadas no presentan residuos en los granos de maní en la cosecha.
\end{abstract}

Palabras clave: Arachis hypogeae L.; Plaga del suelo; Residuo de insecticida.

\section{Introdução}

Os percevejos-cavadores, como são conhecidos, são fitófagos e a maioria das espécies provavelmente polífagas (FROESCHNER, 1960; LIS et al., 2000). O grupo tem sido considerado de pouca importância econômica (LIS et al. 2000), no entanto os danos às culturas na região Neotropical têm crescido nos últimos anos (OLIVEIRA et al., 2000; OLIVEIRA et al., 2013).

Na área cultivada com amendoim no Estado de São Paulo, tem-se observado um aumento expressivo de percevejo-preto, Cyrtomenus mirabilis (Hemiptera: Cydnidae), na qual ninfas e adultos ao se alimentarem das vagens, inserem o estilete de seu aparelho bucal, atingindo os grãos em 
desenvolvimento, tornando-os manchados e impróprios para comercialização (GALLO et al., 2002).

Para detecção de sua presença na área, podem-se utilizar armadilhas luminosas ou armadilhas de queda, principalmente no início de desenvolvimento das plantas quando ainda não se tem vagens em estágios mais avançados de maturação (MICHELOTTO et al., 2019).

Adultos de C. mirabilis e suas fases jovens vivem no solo, incluindo sua oviposição (RIIS et al., 2005). Em função desse hábito subterrâneo, o controle de C. mirabilis torna-se difícil de ser realizado. A utilização de inseticidas no tratamento de sementes nas dosagens recomendadas não tem sido suficiente para redução da população e as pulverizações foliares realizadas para controle de pragas da parte aérea parecem não ter efeito sobre a mesma.

Estudos relacionados ao controle deste inseto foram realizados na década de 1960 com inseticidas altamente tóxicos e não utilizados atualmente (CALCAGNOLO e DE TELLA, 1965). Nos Estados Unidos, uma das poucas opções de inseticida para controle de Pangaeus bilineatus (Say) que causa dano similar em amendoim é o clorpirifós (Lorsban® $15 \mathrm{G}$ ) na fórmula granulada, aplicado em faixas sobre as plantas de amendoim (CHAPIN e THOMAS, 2003).

Recentemente, trabalhos realizados em laboratório mostraram que alguns inseticidas podem ser eficientes para controle tanto de ninfas quanto de adultos do percevejo-preto em amendoim (RINCÃO et al., 2019). No entanto, estes resultados precisam ser realizados em condições de campo e os resíduos deles em amendoim também precisam ser acompanhados.

Dessa forma, o objetivo deste trabalho foi avaliar a eficiência de inseticidas no controle de ninfas e adultos do percevejo-preto e na redução dos danos nos grãos ocasionados pelos mesmos além de avaliar os resíduos dos ingredientes ativos dos inseticidas nos grãos de amendoim. 


\section{Material e métodos}

Foram instalados dois experimentos na safra 2019/20 em área experimental da Apta/Polo Centro Norte em Pindorama e do Centro de Cana do IAC em Ribeirão Preto. Em função da dificuldade de se identificar sua presença na área, os locais foram escolhidos baseados no histórico de ocorrência em safras anteriores.

O delineamento experimental adotado foi em blocos casualizados, com 4 repetições. Os tratamentos são apresentados na Tabela 1.

Tabela 1. Inseticidas, ingredientes ativos, dosagem e época/local de aplicação para controle do percevejo-preto. Safra 2019/20.

\begin{tabular}{|c|c|c|c|c|}
\hline Tratamento & Ingrediente Ativo & $\begin{array}{l}\text { Dosagem } \\
\text { Comercial }\end{array}$ & $\begin{array}{c}\text { Dosagem } \\
\text { de Fipronil / ha } \\
\end{array}$ & $\begin{array}{c}\text { Época/Local de } \\
\text { aplicação }\end{array}$ \\
\hline Testemunha & - & - & 0 & - \\
\hline Standak $\circledast$ Top & $\begin{array}{c}\text { Piraclostrobina + } \\
\text { Tiofanato Metílico + } \\
\text { Fipronil }\end{array}$ & $\begin{array}{c}200 \mathrm{~mL} / 100 \mathrm{~kg} \\
\text { de sementes }\end{array}$ & $75 \mathrm{~g}$ & $\begin{array}{l}\text { Tratamento de } \\
\text { sementes }\end{array}$ \\
\hline Regent $\circledast$ Duo & $\begin{array}{c}\text { Fipronil + } \\
\text { Alfacipermetrina }\end{array}$ & $500 \mathrm{~mL} \mathrm{ha}^{-1}$ & $90 \mathrm{~g}$ & $\begin{array}{c}\text { Sulco na } \\
\text { semeadura }\end{array}$ \\
\hline Regent® Duo & $\begin{array}{c}\text { Fipronil + } \\
\text { Alfacipermetrina }\end{array}$ & $600 \mathrm{~mL} \mathrm{ha}^{-1}$ & $108 \mathrm{~g}$ & $\begin{array}{c}\text { Sulco na } \\
\text { semeadura }\end{array}$ \\
\hline Standak® Top & $\begin{array}{c}\text { Piraclostrobina + } \\
\text { Tiofanato Metílico + } \\
\text { Fipronil }\end{array}$ & $\begin{array}{c}300 \mathrm{~mL} / 100 \mathrm{~kg} \\
\text { de sementes }\end{array}$ & $112,5 \mathrm{~g}$ & $\begin{array}{l}\text { Tratamento de } \\
\text { sementes }\end{array}$ \\
\hline Regent® Duo & $\begin{array}{c}\text { Fipronil + } \\
\text { Alfacipermetrina }\end{array}$ & $800 \mathrm{~mL} \mathrm{ha}^{-1}$ & $144 \mathrm{~g}$ & $\begin{array}{c}\text { Sulco na } \\
\text { semeadura }\end{array}$ \\
\hline Standak $\circledast$ Top & $\begin{array}{c}\text { Piraclostrobina + } \\
\text { Tiofanato Metílico + } \\
\text { Fipronil }\end{array}$ & $\begin{array}{c}400 \mathrm{~mL} / 100 \mathrm{~kg} \\
\text { de sementes }\end{array}$ & $150 \mathrm{~g}$ & $\begin{array}{l}\text { Tratamento de } \\
\text { sementes }\end{array}$ \\
\hline $\begin{array}{c}\text { Regent® Duo } \\
+ \\
\text { Standak } \AA \text { Top }\end{array}$ & $\begin{array}{c}\text { Fipronil + } \\
\text { Alfacipermetrina } \\
+ \\
\text { Piraclostrobina + } \\
\text { Tiofanato Metílico + } \\
\text { Fipronil }\end{array}$ & $\begin{array}{c}500 \mathrm{~mL} \mathrm{ha}_{+}^{-1} \\
200 \mathrm{~mL} / 100 \mathrm{~kg} \\
\text { de sementes }\end{array}$ & $165 \mathrm{~g}$ & $\begin{array}{c}\text { Sulco na } \\
\text { semeadura } \\
+ \\
\text { Tratamento de } \\
\text { sementes }\end{array}$ \\
\hline Regent® Duo & $\begin{array}{c}\text { Fipronil + } \\
\text { Alfacipermetrina }\end{array}$ & $1000 \mathrm{~mL} \mathrm{ha}{ }^{-1}$ & $180 \mathrm{~g}$ & $\begin{array}{l}\text { Sulco na } \\
\text { semeadura }\end{array}$ \\
\hline Sabre® & Clorpirifós & $2000 \mathrm{~mL} \mathrm{ha}{ }^{-1}$ & $900 g^{*}$ & $\begin{array}{l}\text { Sulco na } \\
\text { semeadura }\end{array}$ \\
\hline \multicolumn{5}{|c|}{ * $=$ Gramas de Clorpirifós. } \\
\hline
\end{tabular}

Cada parcela foi constituída de quatro linhas de 5 metros de comprimento, com espaçamento entre linhas de $0,9 \mathrm{~m}$. A semeadura foi realizada manualmente e na densidade de 20 sementes por metro, utilizando 
a cultivar IAC OL3. A semente foi previamente tratada com fungicida registrado para controle de doenças redutoras de estande.

A aplicação no sulco de semeadura foi realizada com pulverizador costal elétrico, dotado de ponta de pulverização do tipo leque e com volume de 100 litros ha-1. Já o tratamento de semente com inseticida foi realizado com equipamento laboratorial para tratamento de sementes (Figura 1).
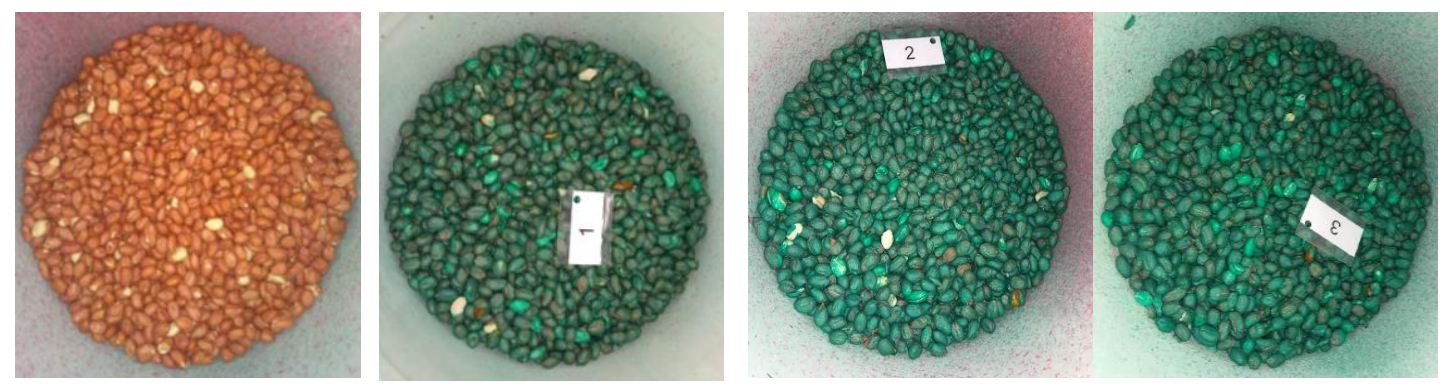

Figura 1. Da esquerda para a direita. Tratamentos de sementes: testemunha sem tratamento; Standak Top nas dosagens $2,0 \mathrm{ml} \mathrm{kg}^{-1}, 3,0 \mathrm{ml} \mathrm{kg}^{-1}$ e 4,0 ml $\mathrm{kg}^{-1}$ de sementes, respectivamente.

Após a emergência das plantas, os experimentos foram conduzidos realizando-se o controle de pragas da parte aérea e de doenças fúngicas foliares de acordo com as recomendações para a cultura (GODOY et al., 2014).

Nos dois locais, aos 130 DAS, para a quantificação dos percevejos no solo nos diferentes tratamentos, foi utilizada a metodologia descrita por Oliveira e Malaguido (2004). Em cada parcela foi aberta uma trincheira de $0,3 \mathrm{~m}$ de comprimento $X 0,3 \mathrm{~m}$ de largura $X 0,15 \mathrm{~m}$ de profundidade, utilizando uma forma de metal. A escavação (retirada das amostras de solo) foi realizada manualmente, com o auxílio de formas e enxadões na profundidade de $15 \mathrm{~cm}$. O solo coletado foi cuidadosamente inspecionado com o auxílio de água e peneiras e os percevejos presentes foram contabilizados e expressos em percevejos por trincheira.

Concomitantemente, foi realizada a amostragem de 3 a 6 plantas por parcela, para avaliação dos danos ocasionados pelos percevejos nos grãos. As vagens foram armazenadas em freezer (temperatura de $\pm 3^{\circ} \mathrm{C}$ ) e posteriormente avaliadas para quantificação do número de grãos 
apresentando sinais de ataque do percevejo e expressos em porcentagem de grãos com sintomas de danos.

Além disso, por ocasião da colheita, uma amostra de 600 gramas de grãos secos ( $7 \%$ de umidade) de cada parcela, foi encaminhada para 0 laboratório JLA Brasil que procedeu a análise de resíduos dos inseticidas avaliados e comparados com os limites máximos de resíduos (LMR) estabelecidos para cada ingrediente ativo.

Os dados de insetos por trincheira e do percentual de grãos com danos visuais foram submetidos à análise de variância pelo teste $\mathrm{F} e$ as médias comparadas pelo teste Tukey a $5 \%$ de probabilidade. A porcentagem de eficiência de controle $(E C)$ e eficiência de redução do dano (ER) foi calculada, conforme Abbott (1925).

\section{Resultados e discussão}

\section{Experimento em Pindorama}

A ocorrência do percevejo-preto em Pindorama foi baixa e irregular. A baixa ocorrência do inseto afetou diretamente o percentual de grãos com sintomas de ataque do percevejo-preto. Os tratamentos apresentaram percentuais de grãos com sintomas de no máximo 1,3\%, o que é extremamente baixo o que impede comparações entre os tratamentos. Assim, os demais parâmetros não foram aqui apresentados.

Esta baixa infestação reforça a necessidade de repetição dos experimentos em um maior número de locais possíveis uma vez que essa praga é de difícil detecção no momento da semeadura.

\section{Experimento em Ribeirão Preto}

Em Ribeirão Preto observou-se uma alta infestação do inseto. $\mathrm{Na}$ ausência de controle, observou-se em média próximo de 30 insetos por trincheira, principalmente ninfas. 
A ocorrência de percevejos por trincheira foi significativamente maior na testemunha, 28,2 e 29,2 respectivamente. Os tratamentos Sabre e Standak Top 200 apresentaram números intermediários, enquanto os demais tratamentos os menores números, com eficiência de controle variando de 82,0\% (Regent Duo 800) a 96,1\% (Standak Top 400 e Standak Top 200 + Regent Duo 500) (Figuras 2).

O percentual de grãos com danos seguiu a mesma tendência observada para o número de insetos. O maior percentual foi observado na testemunha $(41,2 \%)$ e intermediário nos tratamentos Sabre, Standak Top 300 e Standak Top 200 com 25,9\%, 12,2\% e 10,9\% respectivamente (Figura 3). Entre os menos danificados, destaque para os tratamentos Standak Top 400, Standak Top 200 + Regent Duo 500 e Regent Duo 1000, com eficácia de controle dos danos acima de $87 \%$.

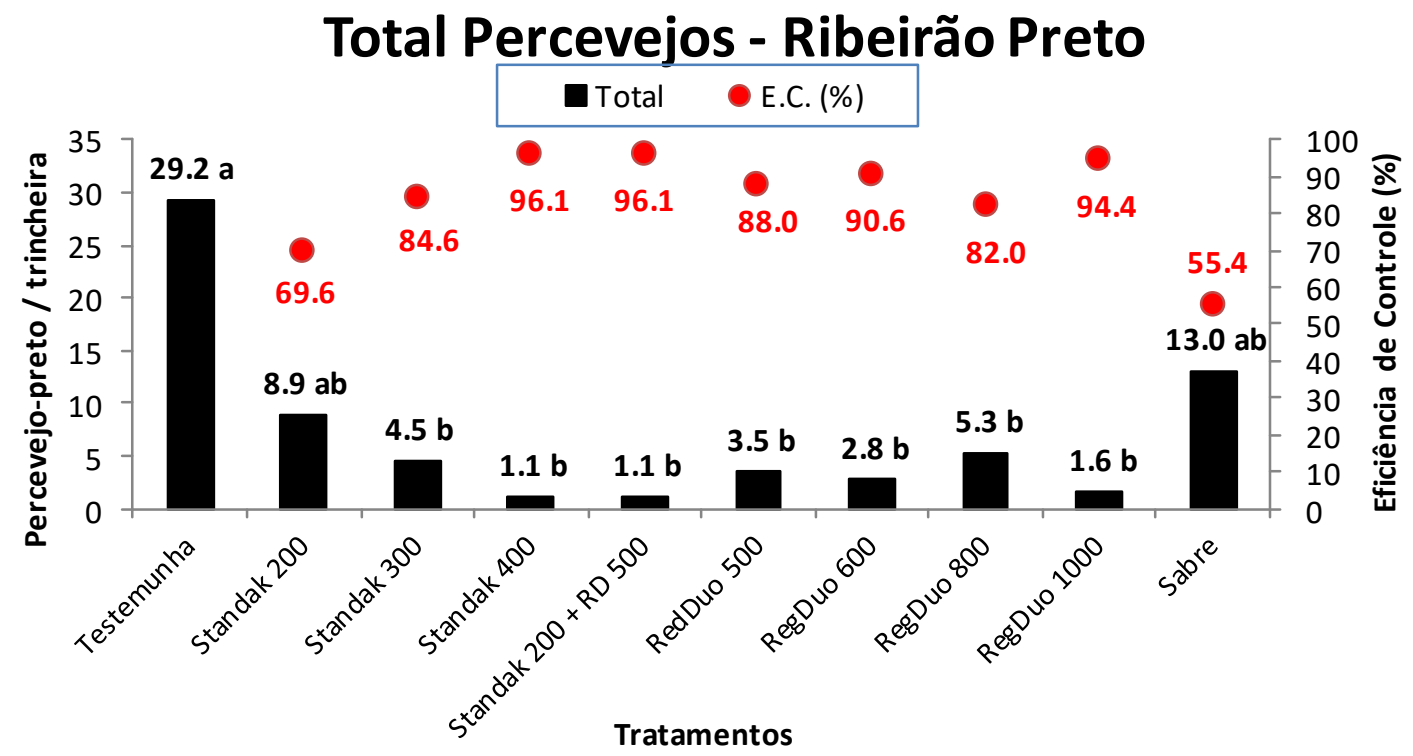

Figura 2. Número total de percevejos por trincheira e eficiência de controle em função dos tratamentos adotados em Ribeirão Preto, SP. Médias seguidas de letras diferentes nas colunas diferem entre si pelo teste de Tukey a 5\% de probabilidade. $\left(F=5,84^{* *} ; C V=48,99 ;{ }^{* *}=\right.$ significativo a $1 \%$ de probabilidade $)$.

Os resíduos das moléculas inseticidas utilizadas nos experimentos foram avaliados nos grãos de amendoim em todos os tratamentos, inclusive no experimento realizado em Pindorama. Nenhuma das moléculas nas dosagens utilizadas foi detectada nas análises, ou seja, todas apresentaram resíduo $<0,001 \mathrm{mg} \mathrm{kg}^{-1}$, mínimo detectável. 


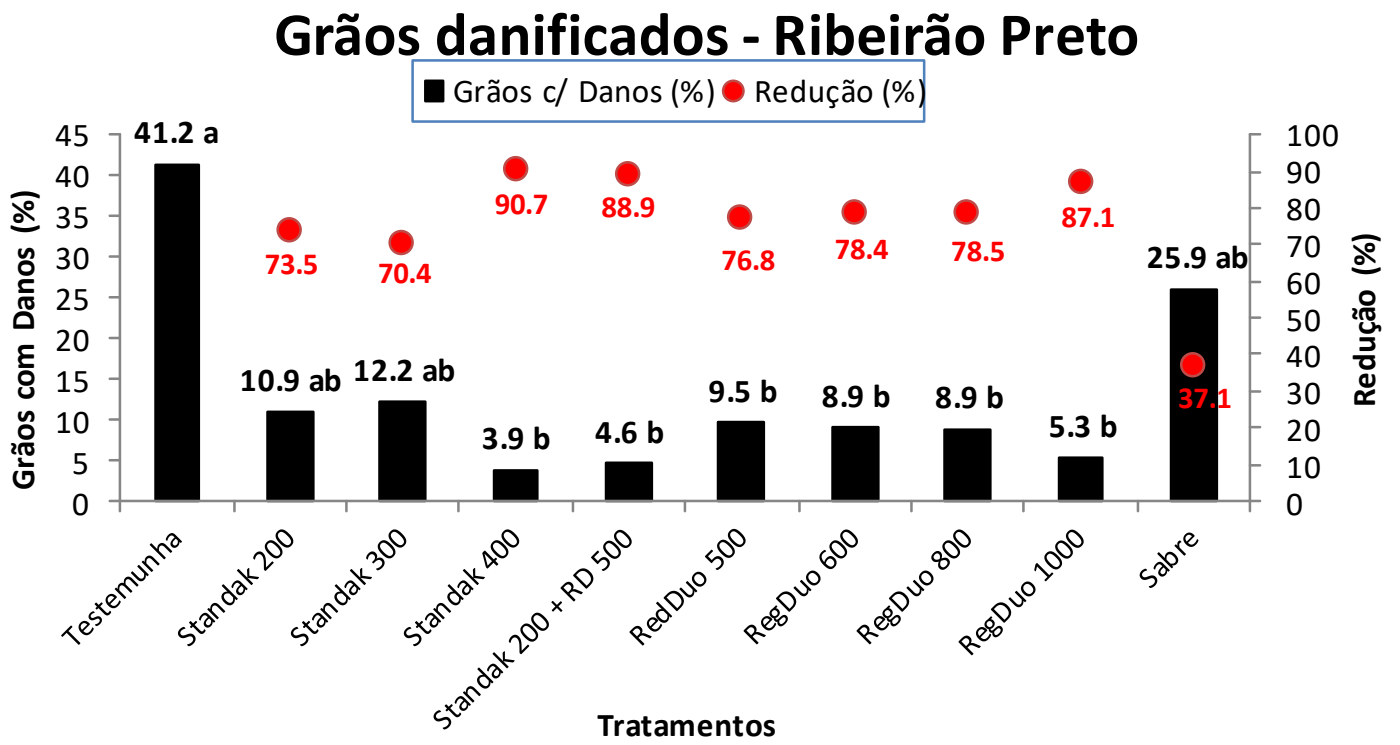

Figura 3. Média de grãos com danos (\%) do percevejo-preto e redução (\%) em relação à testemunha em função dos tratamentos adotados em Ribeirão Preto, SP. Médias seguidas de letras diferentes nas colunas diferem entre si pelo teste de Tukey a $5 \%$ de probabilidade. $\left(F=3,82^{* *}\right.$; $C V=78,67 ;{ }^{* *}=$ significativo a $1 \%$ de probabilidade).

No entanto, novos experimentos devem ser realizados visando a confirmação dos resultados e, portanto, obter maior segurança em relação à possibilidade de uso do inseticida no controle do percevejo-preto em amendoim.

\section{Conclusões}

Em Pindorama observou-se baixa infestação do inseto impedindo comparações entre os tratamentos. Os tratamentos Standak Top $(3,0 \mathrm{~mL} \mathrm{~kg}$ ${ }^{1}$ de sementes), Standak Top (4,0 $\mathrm{mL} \mathrm{kg}^{-1}$ de sementes) e Standak Top (2,0 $\mathrm{mL} \mathrm{kg}^{-1}$ de sementes) associado ao Regent Duo (500 ml ha-1) são eficientes para controle e redução dos danos do percevejo-preto. Os produtos nas doses e nas condições avaliadas não apresentam resíduo dos princípios ativos nos grãos de amendoim depois de colhidos.

\section{Agradecimentos}

Os autores agradecem ao CNPq pela bolsa de produtividade DT ao último autor e às Empresas Amenco, Balsamo, Beatrice, Casul, Copercana, 
Coplana, Mars Brasil e Terra Nuts pelo aporte financeiro ao projeto através da Fundag. 


\section{Referências}

ABBOTT, W.S. A method of computing the effectiveness of an insecticide. Journal Economic Entomology, v.18, n.1, p.265-266. 1925.

CALCAGNOLO, G.; de TELLA, R. Resultados dos experimentos de combate ao Cyrtomenus mirabilis Perty, 1834 - percevejo preto da raiz do amendoinzeiro. O Biológico, v.31, p.21-31, 1965.

CHAPIN, Jay W.; THOMAS, James S. Burrower bugs (Heteroptera: Cydnidae) in peanut: seasonal species abundance, tillage effects, grade reduction effects, insecticide efficacy, and management. Journal of Economic Entomology, v.96, n.4, p.1142-1152, 2003. https://doi.org/10.1603/0022-0493-96.4.1142

FROESCHNER, R.C. Cydnidae of the Western Hemisphere. Proceedings of the United States National Museum, v.111, p.337-680, 1960.

GALLO, D.; NAKANO, O.; NETO, S.S.; CARVALHO, R.P.L.; BAPTISTA, G.C.; FILHO, E.B.; PARRA, J.R.P.; ZUCCHI, R.A.; ALVES, S.B.; VENDRAMIM, J.D.; MARCHINI, L.C.; LOPES, J.R.S.; OMOTO, C. Entomologia agrícola. 10.ed. Piracicaba: FEALQ, 2002. 920p.

GODOY, I.J.; BOLONHEZI, D.; MICHELOTTO, M.D.; FINOTO, E.L.; KASAI, F.S.; FREITAS, R.S. Amendoim - Arachis hypogaea L. In: AGUIAR, A.T.E.; GONÇALVES, C.; PATERNIANI M.E.A.G.Z.; TUCCI, M.L.S.; CASTRO, C.E.F. (Eds.). Instruções agrícolas para as principais culturas econômicas. $7^{a}$ Ed. rev. e atual. Campinas: Instituto Agronômico, 2014. p. 22-27p. (Boletim IAC, № 200).

LIS, J.A.; BECKER, M.; SCHAEFER, C.W. Burrower bugs (Cydnidae). In: SCHAEFER, C.W.; PANIZZI, A.R. (eds) Heteroptera of Economic Importance, CRC Press, London, New York, Washington, p 405-419. 2000.

MICHELOTTO, M.D.; RINCAO, R.O.; BOLONHEZI, D.; FREITAS, R.S. de; FERRAZ, M.; GODOY, I.J. de, SCHWERTNER, C.F. Flutuação populacional do percevejo-preto, Cyrtomenus mirabilis (Perty, 1836) (Hemiptera: Cydnidae) com o uso de armadilhas em amendoim. Brazilian Journal of $\begin{array}{llllll}\text { Development, v. } 5, \quad \text { n. } 10, & \text { p. 22529-22540, } 2019 .\end{array}$ https://doi.org/10.34117/bjdv5n10-371

OLIVEIRA, L.J., MALAGUIDO, A.B. Flutuação e distribuição vertical da população do percevejo castanho da raiz, Scaptocoris castanea Perty (Hemiptera: Cydnidae), no perfil do solo em áreas produtoras de soja nas regiões Centro-Oeste e Sudeste do Brasil. Neotropical Entomology, v.33, p.283-291, 2000. https://doi.org/10.1590/S1519-566X2004000300002

OLIVEIRA, L.J.; MALAGUIDO, A.B.; NUNES Jr, J.; CORSO, I.C.; DE ANGELIS, S., FARIAS, L.C.; HOFFMANN-CAMPO, C.B.; LANTMANN, A.. Percevejos castanhos da raiz em sistemas de produção de soja. Embrapa-Soja, Londrina, PR, Brasil. 2000. 44p. 
OLIVEIRA LJ, ROGGIA S, SALVADORI JR, ÁVILA CJ, FERNANDES PM, OLIVEIRA CM. Insetos que atacam raízes e nódulos da soja. HOFFMANNCAMPO, CB; CORRÊA-FERREIRA, BS; MOSCARDI, F. Soja: manejo integrado de insetos e outros artrópodes-praga, cap, 2, 75-144, 2013.

RINCÃO, R.O.; FERRAZ, M.; POLLI JUNIOR, P.C.; FREITAS, R.S. de; GODOY, I.J. de; MICHELOTTO, M.D. Eficiência de inseticidas no controle de percevejo-preto, Cyrtomenus mirabilis (Perti, 1830) em condições de laboratório. Em: Anais do XVI Encontro Sobre a Cultura do Amendoim. Campinas: Galoá. 2019.

Disponível em: <https://proceedings.science/encontro-amendoim-2019/papers/eficiencia-deinseticidas-no-controle-de-percevejo-preto--cyrtomenus-mirabilis--perti-1830--em-condicoes-de-laboratorio> Acesso em: 16 jul. 2020.

RIIS, L.; BELOTTI, A.C.; ARIAS, B. Bionomics and population growth statistics of Cyrtomenus bergi (Hemiptera: Cydnidae) on different host plants. Florida Entomologist, v.88, p.1-10, 2005. https://doi.org/10.1093/jee/96.6.1905 